\title{
An intrusive hybrid method for discontinuous two-phase flow under uncertainty
}

Per Pettersson, Gianluca Iaccarino and Jan Nordström

\section{Linköping University Post Print}

\section{Tweet}

N.B.: When citing this work, cite the original article.

Original Publication:

Per Pettersson, Gianluca Iaccarino and Jan Nordström, An intrusive hybrid method for discontinuous two-phase flow under uncertainty, 2013, Computers \&amp; Fluids, (86), 228239.

http://dx.doi.org/10.1016/j.compfluid.2013.07.009

Copyright: Elsevier

http://www.elsevier.com/

Postprint available at: Linköping University Electronic Press

http://urn.kb.se/resolve?urn=urn:nbn:se:liu:diva-96372 


\title{
An intrusive hybrid method for discontinuous two-phase flow under uncertainty
}

\author{
Per Pettersson ${ }^{\mathrm{a}, \mathrm{b}, *}$, Gianluca Iaccarino ${ }^{\mathrm{a}}$, Jan Nordström ${ }^{\mathrm{c}}$ \\ ${ }^{a}$ Institute for Computational and Mathematical Engineering, Stanford University, \\ Stanford, CA 94305, USA. \\ ${ }^{b}$ Department of Information Technology, Uppsala University, SE-75105 Uppsala, Sweden \\ ${ }^{c}$ Department of Mathematics, Linköping University, SE-58183 Linköping, Sweden
}

\section{Abstract}

An intrusive stochastic projection method for two-phase time-dependent flow subject to uncertainty is presented. Numerical experiments are carried out assuming uncertainty in the location of the physical interface separating the two phases, but the framework generalizes to uncertainty with known distribution in other input data. Uncertainty is represented through a truncated multiwavelet expansion.

We assume that the discontinuous features of the solution are restricted to computational subdomains and use a high-order method for the smooth regions coupled weakly through interfaces with a robust shock capturing method for the non-smooth regions.

The discretization of the non-smooth region is based on a generalization of the HLL flux, and have many properties in common with its deterministic counterpart. It is simple and robust, and captures the statistics of the shock.

\footnotetext{
${ }^{*}$ Corresponding author. Address: 488 Escondido Mall, Stanford University, Stanford, CA 94305-3024, USA. Phone: +16507216565

Email addresses: massperp@stanford.edu (Per Pettersson ), jops@stanford.edu (Gianluca Iaccarino), jan.nordstrom@liu.se (Jan Nordström)
} 
The discretization of the smooth region is carried out with high-order finitedifference operators satisfying a summation-by-parts property.

Keywords: Uncertainty quantification, Stochastic Galerkin Method, Hybrid scheme, Summation by parts operators

\section{Introduction}

Physical models in computational fluid dynamics can be extended with stochastic models to represent uncertainty in governing equations or input parameters, including e.g. boundary and initial conditions, geometry and rates of reaction, diffusion and advection. One can distinguish between nonintrusive methods, where existing deterministic numerical solvers are called with a range of input values, and intrusive methods, resulting in a modified problem that is larger than the original deterministic problem, but only needs to be solved once. Despite the increased complexity of the reformulated problem, it has the potential to result in reduced computational cost compared to that of non-intrusive methods, such as Monte Carlo methods or stochastic numerical integration methods.

A stochastic two-phase problem in one spatial dimension is investigated as a first step towards developing an intrusive method for shock-bubble interaction with generic uncertainty in the input parameters. So et al [1] investigated a two-dimensional two-phase problem subject to uncertainty in bubble deformation and contamination of the gas bubble, based on the experiments in [2]. The eccentricity of the elliptic bubble and the ratio of air-helium of the bubble were assumed to be random variables, and quantities of interest were obtained by numerical integration in the stochastic range (stochastic 
collocation). Previous work on uncertainty quantification for multi-phase problems include petroleum reservoir simulations with stochastic point collocation methods where deterministic flow solvers are evaluated at stochastic collocation points [3] and Karhunen-Loève expansions combined with perturbation methods [4].

We assume uncertainty in the location of the material interface, which requires a stochastic representation of all flow variables. Stochastic quantities are represented as generalized chaos series, that could be either global as in the case of generalized polynomial chaos (gPC)[5], or localized, see e.g. [6]. For robustness, we use a generalized chaos expansion with multiwavelets to represent the solution in the stochastic dimension [7]. It should be noted that this basis is global, so the method is fully intrusive. However, the basis is hierarchically localized in the sense that multiwavelets belonging to the same resolution level are grouped into families with non-overlapping support. These features makes it suitable for approximating discontinuities in the stochastic space without the emergence of spurious oscillations that occur in the case of global polynomial bases.

The stochastic Galerkin method is applied to the stochastic two-phase formulation, resulting in a finite-dimensional deterministic system that shares many properties with the original deterministic problem. The regularity properties of the stochastic problem are essential in the design of an appropriate numerical method. Chen et al studied the steady-state inviscid Burgers' equation with a source term [8]. We used a similar approach for the inviscid Burgers' equation with uncertain boundary conditions and also analyzed the regularity of low-order stochastic Galerkin approximations of 
the problem [9]. Schwab and Tokareva analyzed regularity of scalar hyperbolic conservation laws and a linearized version of the Euler equations with uncertain initial profile [10]. In this paper, we analyze smoothness of the stochastic two-phase system.

The stochastic Galerkin problem is hyperbolic. This generalized and extended two-phase problem is solved with a hybrid method coupling the continuous phase region with the discontinuous phase region through a numerical interface. The non-smooth region is solved with the HLL-flux and MUSCL-reconstruction in space. The minmod flux limiter is employed in the numerical experiments displayed below.

Finite-difference operators in summation-by-parts (SBP) form are used for the high-order spatial discretization. A symmetrized problem formulation that generalizes the energy estimates in [11] for the Euler equations is used for the stochastic Galerkin system. The coupling between the different solution regions is performed with a weak imposition of the interface conditions through an interface using a penalty technique [12]. A fourth order Runge-Kutta method is used for the integration in time.

\section{Representation of uncertainty}

The polynomial chaos framework for uncertainty quantification introduced in [13] and generalized in [5] is used to represent uncertainty in the input parameters of the governing equations.

Let $\omega$ be an outcome of a probability space $(\Omega, \mathcal{A}, \mathcal{P})$, with event space $\Omega, \sigma$-algebra $\mathcal{A}$, and probability measure $\mathcal{P}$. Let $\boldsymbol{\xi}=\left\{\xi_{j}(\omega)\right\}_{j=1}^{N}$ be a set of $N$ i.i.d. random variables for $\omega \in \Omega$. Each random variable $\xi_{j}$ is a mapping 
from the event space to $\mathbb{R}$. For the cases presented here, $N=1$ i.e. a single source of uncertainty is assumed but the framework can be generalized to multiple sources of uncertainty.

Consider a generalized chaos basis $\left\{\psi_{i}(\xi)\right\}_{i=0}^{\infty}$ spanning the space of second order (i.e. finite variance) random processes on this probability space. The basis functions are assumed to be orthonormal,

$$
\left\langle\psi_{i} \psi_{j}\right\rangle=\int_{\Omega} \psi_{i}(\xi) \psi_{j}(\xi) d \mathcal{P}(\xi)=\delta_{i j},
$$

where $\delta_{i j}$ is the Kronecker delta. Second order random fields $u(x, t, \xi)$ can be expressed as

$$
u(x, t, \xi)=\sum_{i=0}^{\infty} u_{i}(x, t) \psi_{i}(\xi) .
$$

Independent of the choice of orthogonal basis $\left\{\psi_{i}\right\}_{i=0}^{\infty}$, we can express mean and variance of $u(x, t, \xi)$ as

$$
E(u(x, t, \xi))=\int_{\Omega} u(x, t, \xi) d \mathcal{P}(\xi)=u_{0}(x, t),
$$

and

$$
\operatorname{Var}(u(x, t, \xi))=\int_{\Omega}(u(x, t, \xi)-E(u(x, t, \xi)))^{2} d \mathcal{P}(\xi)=\sum_{i=1}^{\infty} u_{i}^{2}(x, t),
$$

respectively.

In the context of the stochastic Galerkin method, (1) is truncated to $M+1$ terms, and we set

$$
u(x, t, \xi) \approx \sum_{i=0}^{M} u_{i}(x, t) \psi_{i}(\xi) .
$$

The truncated generalized chaos solution converges in the $L_{2}(\Omega, \mathcal{P})$ norm to the exact solution as $M \rightarrow \infty$. 


\subsection{Multiwavelet expansion}

Hyperbolic problems exhibit sharp gradients and shocks, for which polynomial representations are prone to fail, see e.g. [14, 15]. For robustness, we follow the approach of [16] and use piecewise polynomial multiwavelets $(\mathrm{MW})$, defined on sub-intervals of $[-1,1]$. The construction of a truncated MW basis follows the algorithm in [17].

Wavelets are defined hierarchically on different resolution levels, representing successively finer features of the solution with increasing resolution. They have non-overlapping support within each resolution level, and in this sense they are localized. Still, the basis is global due to the overlapping support of wavelets belonging to different resolution levels. Piecewise constant wavelets, denoted Haar wavelets, do not exhibit spectral convergence, but avoid the Gibbs phenomenon in the proximity of discontinuities in the stochastic dimension.

Starting with the space $\mathbf{V}_{N_{p}}$ of polynomials of degree at most $N_{p}$ defined on the interval $[-1,1]$, the construction of multiwavelets aims at finding a basis of piecewise polynomials for the orthogonal complement of $\mathbf{V}_{N_{p}}$ in the space $\mathbf{V}_{N_{p}+1}$ of polynomials of degree at most $N_{p}+1$. Merging the bases of $\mathbf{V}_{N_{p}}$ and that of the orthogonal complement of $\mathbf{V}_{N_{p}}$ in $\mathbf{V}_{N_{p}+1}$, we

obtain a piecewise polynomial basis for $\mathbf{V}_{N_{p}+1}$. Continuing the process of finding orthogonal complements in spaces of increasing degree of piecewise polynomials, leads to a basis for $L_{2}([-1,1])$.

We first introduce a smooth polynomial basis on $[-1,1]$. Let $\left\{L e_{i}(\xi)\right\}_{i=0}^{\infty}$ 
be the normalized Legendre polynomials, defined recursively by

$$
\begin{aligned}
& L e_{i+1}(\xi)=\sqrt{2 i+3}\left(\frac{\sqrt{2 i+1}}{i+1} \xi L e_{i}(\xi)-\frac{i}{(i+1) \sqrt{2 i-1}} L e_{i-1}(\xi)\right), i \geq 1, \\
& L e_{0}(\xi)=1, \quad L e_{1}(\xi)=\sqrt{3} \xi .
\end{aligned}
$$

The set $\left\{L e_{i}(\xi)\right\}_{i=0}^{N_{p}}$ is an orthonormal basis for $\mathbf{V}_{N_{p}}$.

Following the algorithm by Alpert [17], we construct a set of mother wavelets $\left\{\psi_{i}^{W}(\xi)\right\}_{i=0}^{N_{p}}$ defined on the domain $\xi \in[-1,1]$, where

$$
\psi_{i}^{W}(\xi)= \begin{cases}p_{i}(\xi) & -1 \leq \xi<0 \\ (-1)^{N_{p}+i+1} p_{i}(\xi) & 0 \leq \xi<1 \\ 0 & \text { otherwise }\end{cases}
$$

where $p_{i}(\xi)$ is an $i^{\text {th }}$ order polynomial. By construction, the set of wavelets $\left\{\psi_{i}^{W}(\xi)\right\}_{i=0}^{N_{p}}$ are orthogonal to all polynomials of order at most $N_{p}$, hence the wavelets are orthogonal to the set $\left\{L e_{i}(\xi)\right\}_{i=0}^{N_{p}}$ of Legendre polynomials of order at most $N_{p}$. Based on translations and dilations of (2), we get the wavelet family

$\psi_{i, j, k}^{W}(\xi)=2^{j / 2} \psi_{i}^{W}\left(2^{j} \xi-k\right), \quad i=0, \ldots, N_{p}, \quad j=0,1, \ldots, \quad k=0, \ldots, 2^{j-1}$

Let $\psi_{m}(\xi)$ for $m=0, \ldots, N_{p}$ be the set of Legendre polynomials up to order $N_{p}$, and concatenate the indices $i, j, k$ into $m=\left(N_{p}+1\right)\left(2^{j}+k-1\right)+i$ so that $\psi_{m}(\xi) \equiv \psi_{i, j, k}^{W}(\xi)$ for $m>N_{p}$. With the MW basis $\left\{\psi_{m}(\xi)\right\}_{m=0}^{\infty}$ we can represent any random variable $u(x, t, \xi)$ with finite variance as

$$
u(x, t, \xi)=\sum_{m=0}^{\infty} u_{m}(x, t) \psi_{m}(\xi)
$$

which is of the form (1). In the computations, we truncate the MW series both in terms of the piecewise polynomial order $N_{p}$ and the resolution level 
$N_{r}$. With the index $j=0, \ldots, N_{r}$, we retain $M+1=\left(N_{p}+1\right) 2^{N_{r}}$ terms of the MW expansion.

\section{Two-phase flow problem}

We assume two phases with volume fractions $\alpha$ and $\beta=1-\alpha$ on the domain $x \in[0,1]$, governed by the advection equation

$$
\frac{\partial \alpha}{\partial t}+v^{\prime}(x, t) \frac{\partial \alpha}{\partial x}=0,
$$

where we let $v^{\prime}(x, t)=v(x, t)$ be the advective velocity obtained from the conservative Euler system below. The Euler equations determine the conservation of masses $\alpha \rho_{\alpha}$ and $\beta \rho_{\beta}$, momentum $\rho v$, and total energy $E$ of the two phases through

$$
\frac{\partial u}{\partial t}+\frac{\partial f}{\partial x}=0
$$

where

$$
u=\left[\begin{array}{c}
\alpha \rho_{\alpha} \\
\beta \rho_{\beta} \\
\rho v \\
E
\end{array}\right], \quad f=\left[\begin{array}{c}
\alpha \rho_{\alpha} v \\
\beta \rho_{\beta} v \\
\rho v^{2}+p \\
(E+p) v
\end{array}\right] .
$$

We assume that the pressure $p$ is given by the perfect gas equation of state for two phases

$$
p=(\gamma-1)\left(E-\frac{1}{2} \rho v^{2}\right), \quad \gamma=\frac{1}{\frac{\alpha}{\gamma_{\alpha}}+\frac{\beta}{\gamma_{\beta}}},
$$

where $\gamma$ denotes the ratio of specific heats. The total density is given by $\rho=\alpha \rho_{\alpha}+\beta \rho_{\beta}$. Note that the sum of the first and second equations of (4) is the standard mass conservation of the Euler equations. Thus, an 
equivalent formulation is the Euler equations supplemented with an extra mass conservation equation for one of the phases $\alpha$ and $\beta$.

We investigate the Riemann problem defined by the initial conditions

$$
\left(\alpha, \alpha \rho_{\alpha}, \beta \rho_{\beta}, \rho v, E\right)^{T}=\left\{\begin{array}{ll}
(1,1,0,0,2.5)^{T} & x<x_{0}+\xi \\
(0,0,0.125,0,0.25)^{T} & x>x_{0}+\xi
\end{array},\right.
$$

where $\xi$ is a parametrization of the measured or modeled uncertainty in the initial membrane location. Despite the seemingly simple nature of the initial condition, the MW series of the initial condition has an infinite number of non-zero terms. Thus, stochastic truncation error is an issue already at $t=0$. Initial functions are obtained by projection of (6) onto the basis functions $\psi_{i}(\xi)$, for $i=0,1, \ldots, M$ where $M$ is some integer to be chosen.

The stochastic Galerkin formulation of the two-phase problem is obtained by multiplying (3) and (4) by each one of the basis functions $\psi_{i}(\xi)$, and integrating with respect to the probability measure $\mathcal{P}$ over the range of $\xi$. The MW expansion is truncated to $M+1$ terms and we get the systems for the MW coefficients

$$
\begin{aligned}
& \frac{\partial}{\partial t} \alpha_{k}+\sum_{i=0}^{M} \sum_{j=0}^{M} v_{i} \frac{\partial}{\partial x} \alpha_{j}\left\langle\psi_{i} \psi_{j} \psi_{k}\right\rangle=0, \quad k=0, \ldots, M, \\
& \beta_{k}=\delta_{k 0}-\alpha_{k}, \quad k=0, \ldots, M,
\end{aligned}
$$

and

$$
\frac{\partial}{\partial t}\left[\begin{array}{c}
\left(\alpha \rho_{\alpha}\right)_{k} \\
\left(\beta \rho_{\beta}\right)_{k} \\
(\rho v)_{k} \\
E_{k}
\end{array}\right]+\frac{\partial}{\partial x}\left[\begin{array}{c}
\sum_{i=0}^{M} \sum_{j=0}^{M}\left(\alpha \rho_{\alpha}\right)_{i} v_{j}\left\langle\psi_{i} \psi_{j} \psi_{k}\right\rangle \\
\sum_{i=0}^{M} \sum_{j=0}^{M}\left(\beta \rho_{\beta}\right)_{i} v_{j}\left\langle\psi_{i} \psi_{j} \psi_{k}\right\rangle \\
\sum_{i=0}^{M} \sum_{j=0}^{M}(\rho v)_{i} v_{j}\left\langle\psi_{i} \psi_{j} \psi_{k}\right\rangle+p_{k} \\
\sum_{i=0}^{M} \sum_{j=0}^{M}\left(E_{i}+p_{i}\right) v_{j}\left\langle\psi_{i} \psi_{j} \psi_{k}\right\rangle
\end{array}\right]=0, \quad k=0, \ldots, M
$$


MW expansions for e.g. the pressure can be updated from the MW of the conservative variables, and then be inserted into the fluxes. We use a pseudospectral approximation of high-order stochastic products for the pressure update. In the computation of e.g. the order $M$ product $z(\xi)=\sum_{k=0}^{M} z_{k} \psi_{k}$ of three stochastic variables $a(\xi), b(\xi), c(\xi)$, for $k=0, \ldots, M$, we use the approximation

$$
\begin{array}{r}
z_{k}=(a(\xi) b(\xi) c(\xi))_{k}=\left\langle\left(\sum_{i=0}^{M} a_{i} \psi_{i}(\xi)\right)\left(\sum_{j=0}^{M} b_{j} \psi_{j}(\xi)\right)\left(\sum_{l=0}^{M} c_{l} \psi_{l}(\xi)\right) \psi_{k}(\xi)\right\rangle \\
=\sum_{i=0}^{M} \sum_{j=0}^{M} \sum_{l=0}^{M}\left\langle\psi_{i} \psi_{j} \psi_{k} \psi_{l}\right\rangle a_{i} b_{j} c_{l} \approx \sum_{i=0}^{M} \sum_{m=0}^{M}\left\langle\psi_{i} \psi_{m} \psi_{k}\right\rangle a_{i} \underbrace{\sum_{j=0}^{M} \sum_{l=0}^{M}\left\langle\psi_{j} \psi_{l} \psi_{m}\right\rangle b_{j} c_{l}}_{j=0} \\
\equiv(a *(b * c))_{k}^{M}, \quad(10)
\end{array}
$$

where the pseudo-spectral product $y=a * b$ of order $M$ is defined by

$$
y_{k}^{M}=(a * b)_{k}=\sum_{i=0}^{M} \sum_{j=0}^{M}\left\langle\psi_{i} \psi_{j} \psi_{k}\right\rangle a_{i} b_{j}, \quad k=0, \ldots, M .
$$

In matrix notation, we can express (11) as

$$
y^{M}=A\left(a^{M}\right) b^{M}
$$

where $y^{M}=\left(y_{0}, \ldots, y_{M}\right)^{T}$ is the vector of MW coefficients of $y$ and

$$
\left[A\left(a^{M}\right)\right]_{j+1, k+1}=\sum_{i=0}^{M}\left\langle\psi_{i} \psi_{j} \psi_{k}\right\rangle a_{i} .
$$

By successively applying (12), we obtain approximations of a range of stochastic functions including polynomials, square roots and inverse quantities [18]. 
For general stochastic basis functions and general choices of the order of generalized chaos, the stochastic volume fractions $\alpha$ and $\beta$ cannot be guaranteed to be non-negative. In fact, using first order Legendre polynomial chaos and projecting the initial condition results in $\alpha(x, t=0, \xi)=\alpha_{0}(x, t=$ $0)+\alpha_{1}(x, t=0) \xi, \xi \in \mathcal{U}[-1,1]$. This implies that $\alpha(x, t, \xi)<0$ for some values of $x, t, \xi$. This is clearly undesirable. However, we only use Legendre polynomials for the convergence test of a smooth problem using the method of manufactured solutions in Section 6.1. For the fully discontinuous problem, we use Haar wavelets (piecewise constant multiwavelets, $N_{p}=0$ ) for which the initial function always is physical, no matter the order of wavelet expansion. To see this, we rewrite the stochastic advection system (7) in matrix-vector notation,

$$
\frac{\partial}{\partial t} \alpha^{M}+A\left(v^{M}(x, t)\right) \frac{\partial}{\partial x} \alpha^{M}=0,
$$

where $A($.$) is defined by (13). Assuming Haar wavelets, the matrix A\left(v^{M}(x, t)\right)$ in the stochastic advection system can be diagonalized with constant eigenvectors $y_{k}$, but space- and time-dependent eigenvalues $\lambda_{k}(x, t)$, for $k=$ $0, \ldots, M$. The stochastic Galerkin advection problem then decouples to a set of scalar advection problems,

$$
\frac{\partial}{\partial t} \tilde{\alpha}_{k}+\lambda_{k}(x, t) \frac{\partial}{\partial x} \tilde{\alpha}_{k}=0, \quad \tilde{\alpha}(x, t=0)=\tilde{\alpha}_{k}^{i n i t}(x), \quad k=0, \ldots, M .
$$

where $\tilde{\alpha}_{k}=y_{k}^{T} \alpha$. The solution of the semi-linear advection problem (14) is $\tilde{\alpha}_{k}^{i n i t}\left(r_{k}(x, t)\right)$ where $r_{k}$ is defined by $t=\int_{r_{k}}^{x} \frac{d x^{\prime}}{\lambda_{k}\left(x^{\prime}, t\right)}$. No matter the exact form of $r_{k}$, the solution will never attain values beyond the range of $\tilde{\alpha}_{k}^{\text {init }}$. This implies that the stochastic volume fraction PDE formulation will never yield unphysical values. 


\section{Smoothness properties of the solution}

\subsection{Analytical solution}

The exact solutions to (3) and (4) subject to (6) can be determined analytically, and are discontinuous for all times. The conservation law (4) is a straightforward extension of the Sod test case for shock tube problems and its exact piecewise smooth solution can be found in [19]. The solution consists of five distinct smooth regions (denoted $u_{(L)}, u_{(\exp )}, u_{(2)}, u_{(1)}$ and $u_{(R)}$ ), and the discontinuities may be found at the interfaces between the different regions. Assume that the initial interface location is $x_{0}^{s}=x_{0}+\xi$ as given in (6). We can then express the deterministic solution for any fixed $\xi$ as a piecewise smooth solution, separated by the four spatial points

$$
\begin{aligned}
x_{1}(t, \xi) & =x_{0}+\xi-\sqrt{\gamma \frac{p_{L}}{\rho_{L}}} t \\
x_{2}(t, \xi) & =x_{0}+\xi+\left(v_{2}-\sqrt{\gamma \frac{p_{2}}{\rho_{2}}}\right) t \\
x_{3}(t, \xi) & =x_{0}+\xi+v_{2} t \\
x_{4}(t, \xi) & =x_{0}+\xi+M_{s} t,
\end{aligned}
$$

where $M_{s}$ is the Mach number of the shock.

Any given value of $\xi$ will determine the location of the different regions of piecewise continuous solutions, so the true stochastic solution can be expressed as a function of $\xi$ and the variables of the true deterministic solution. In the $x-t-\xi$-space, all solution discontinuities are defined by triplets $(x, t, \xi)$ satisfying (15) - (18). The solution regions are depicted in Figure 2 (left) for any fixed value of $\xi$. 
For any spatial point $x$, the solution regions can be defined as functions of $\xi$ and $t$. This is shown in Figure 2 (right), where the points in the stochastic dimension separating the different solution regions are given by

$$
\begin{aligned}
& \xi_{1}(x, t)=x-x_{0}+\sqrt{\gamma \frac{p_{L}}{\rho_{L}}} t \\
& \xi_{2}(x, t)=x-x_{0}-\left(v_{2}-\sqrt{\gamma \frac{p_{2}}{\rho_{2}}}\right) t \\
& \xi_{3}(x, t)=x-x_{0}-v_{2} t \\
& \xi_{4}(x, t)=x-x_{0}-M_{s} t .
\end{aligned}
$$

The solution can be written

$$
\begin{aligned}
u(x, t, \xi)=u_{(L)} \mathbb{1}_{\left\{\xi_{1} \leq \xi\right\}}+u_{(\exp )}(x-\xi) & \mathbb{1}_{\left\{\xi_{2} \leq \xi \leq \xi_{1}\right\}}+u_{(2)} \mathbb{1}_{\left\{\xi_{3} \leq \xi \leq \xi_{2}\right\}} \\
& +u_{(1)} \mathbb{1}_{\left\{\xi_{4} \leq \xi \leq \xi_{3}\right\}}+u_{(R)} \mathbb{1}_{\left\{\xi \leq \xi_{\xi_{4}}\right\}}
\end{aligned}
$$

where the indicator function $\mathbb{1}_{\{A\}}$ of a set $A$ is defined by $\mathbb{1}_{\{A\}}(\xi)=1$ if $\xi \in A$ and zero otherwise.

Note that if the range of $\xi$ is bounded, some solution states may not occur with non-zero probability for an arbitrary $x$. The situation shown in Figure 2 (right) requires a sufficiently large range of $\xi$, or, equivalently, that $x$ is sufficiently close to $x_{0}$. The expression (23) is always true however.

\subsection{The stochastic modes}

The solutions of (3) and (4) for fixed values of $\xi$ are discontinuous, but the stochastic modes (multiwavelet coefficients) are continuous. To see this, we proceed from the solution (23) to derive exact expressions for the stochastic modes. We assume that the probability measure $\mathcal{P}$ has a probability density $\tilde{p}$. The $k^{t h}$ mode $u_{k}$ is given by the projection of $(23)$ on $\psi_{k}(\xi)$, 


$$
\begin{gathered}
u_{k}(x, t)=\int_{\Omega} u(x, t, \xi) \psi_{k}(\xi) \tilde{p}(\xi) d \xi=u_{(L)} \int_{\xi_{1}}^{\infty} \psi_{k}(\xi) \tilde{p}(\xi) d \xi \\
+\int_{\xi_{2}}^{\xi_{1}} u_{(\exp )}(x-\xi) \psi_{k}(\xi) \tilde{p}(\xi) d \xi+u_{(2)} \int_{\xi_{3}}^{\xi_{2}} \psi_{k}(\xi) \tilde{p}(\xi) d \xi+u_{(1)} \int_{\xi_{4}}^{\xi_{3}} \psi_{k}(\xi) \tilde{p}(\xi) d \xi \\
+u_{(R)} \int_{-\infty}^{\xi_{4}} \psi_{k}(\xi) \tilde{p}(\xi) d \xi
\end{gathered}
$$

The density $\tilde{p}$ and multiwavelet $\psi_{k}$ are at least piecewise continuous functions, so by $(24) u_{k} \in C^{0}$. Now assume that the parametrization $\xi$ of the uncertainty in the location of the initial discontinuity has a probability density $\tilde{p} \in C^{s}(\mathbb{R})$ for some degree of regularity $s \in \mathbb{N}$. There exists a set $\left\{\psi_{i}\right\}_{i=1}^{\infty}$ of polynomials that are orthogonal with respect to $\tilde{p}$. With this choice of basis functions, we may differentiate (24) with respect to $x$,

$$
\begin{gathered}
\frac{\partial}{\partial x} u_{k}=-u_{(L)} \psi_{k}\left(\xi_{1}\right) \tilde{p}\left(\xi_{1}\right)+u_{\exp }\left(x-\xi_{1}\right) \psi_{k}\left(\xi_{1}\right) \tilde{p}\left(\xi_{1}\right)-u_{\exp }\left(x-\xi_{2}\right) \psi_{k}\left(\xi_{2}\right) \tilde{p}\left(\xi_{2}\right) \\
+\int_{\xi_{2}}^{\xi_{1}} u_{(\exp )}^{\prime}(x-\xi) \psi_{k}(\xi) \tilde{p}(\xi) d \xi+u_{(2)} \psi_{k}\left(\xi_{2}\right) \tilde{p}\left(\xi_{2}\right)-u_{(2)} \psi_{k}\left(\xi_{3}\right) \tilde{p}\left(\xi_{3}\right) \\
\quad+u_{(1)} \psi_{k}\left(\xi_{3}\right) \tilde{p}\left(\xi_{3}\right)-u_{(1)} \psi_{k}\left(\xi_{4}\right) \tilde{p}\left(\xi_{4}\right)+u_{(R)} \psi_{k}\left(\xi_{4}\right) \tilde{p}\left(\xi_{4}\right)
\end{gathered}
$$

where we used that $\partial \xi_{i} / \partial x=1, i=1,2,3,4$. In fact, $u_{k}(x, t)$ as given by (24) is $s+1$ times differentiable in $x$ or $t$ for $t>0$ and $u_{k} \in C^{s+1}$.

Remark 1. Note that the smoothness of $u_{k}$ in $x$ and $t$ ultimately depends on the smoothness of $\tilde{p}$ and the choice of basis functions $\left\{\psi_{i}\right\}_{i=0}^{\infty}$, which are all functions of $\xi$. In contrast, for any fixed value $\xi^{\prime}$, the solution $u\left(x, t, \xi^{\prime}\right)$ is discontinuous in the spatial and temporal dimensions, no matter the smoothness of $\tilde{p}$ and $\left\{\psi_{i}\right\}_{i=0}^{\infty}$. 


\subsection{The stochastic Galerkin solution modes}

We investigated the smoothness properties of the stochastic modes of the original problems problem (3) and (4) above, but in all actual computations we solve the modified stochastic Galerkin approximation (7)-(9). For loworder MW approximations (small $M$ ), the smoothness properties are very different from those derived above. For instance, the $M=0$ approximation is the deterministic two-phase problem with its characteristic discontinuous solution profile. First order gPC approximations using a group of orthogonal polynomials and multiwavelets that are piecewise linear in $\xi$ results in linear combinations of deterministic two-phase problems. In terms of regularity, these problems are clearly equivalent to the deterministic problem. Higher order MW or gPC approximations result in large nonlinear stochastic Galerkin problems that in general cannot be diagonalized into a set of deterministic two-phase problems. Due to their nonlinear nature, we expect these problems to develop discontinuities. However, it is a reasonable assumption that the solution converges to the solution of (4). Hence, we assume that the discontinuities get weaker with the order of gPC expansion so that high-order MW approximations have regularity properties that approach the smoothness properties of the analytical stochastic modes.

We have analyzed smoothness of the particular problem of uncertain initial location of the shock in the Riemann problem (6). An essential feature of the analysis is that for $t>0$, the locations of the discontinuities become stochastic. If this were not the case, the gPC coefficients would not be smooth. Thus, for any given set of initial conditions, smoothness should be analyzed in order to determine about an appropriate numerical method. 
In order to solve (7)-(9) numerically for arbitrary order $M$ of MW expansion (that may vary in space depending on the smoothness of the solution), we need shock-capturing methods that can account for the discontinuities that are expected due to the stochastic truncation. In regions away from the discontinuities, the solution is at least as smooth as the corresponding deterministic problem and high-order methods in combination with smooth polynomial stochastic basis functions are more suitable. In the next section, we present a method which combines high-order and shock-capturing methods for the stochastic Galerkin systems.

\section{Numerical method}

The computational domain is divided into regions of smooth behavior of the solution, and regions of sharp variation. At this stage, these are assumed to be known a priori and do not change with time. Thus there is no need to use a detection algorithm to locate the regions of sharp variation apart from

flux limiters that are applied for smoothing. However, the methodology may be extended to time-dependent regions, see [20]. A fourth-order Runge-Kutta method is used for the time integration.

\subsection{Summation-by-parts operators}

The smooth regions are discretized using a high-order finite difference method based on SBP operators. Boundary conditions are imposed weakly through penalty terms, where the penalty parameters are chosen such that the numerical method is stable. Operators of order $2 n, n \in \mathbb{N}$, in the interior of the domain are combined with boundary closures of order of accuracy $n$. 
The first derivative SBP operator was introduced in [21, 22]. Let $\mathbf{u}$ denote the uniform spatial discretization of $u$. For the first derivative, we use the approximation $u_{x} \approx P^{-1} Q \mathbf{u}$, where subscript $x$ denotes partial derivative and $Q$ satisfies

$$
Q+Q^{T}=\operatorname{diag}(-1,0, \ldots, 0,1) \equiv \tilde{B}
$$

Additionally, $P$ must be symmetric and positive definite in order to define a discrete norm. For the proof of stability, $P$ must be diagonal.

\subsection{HLL Riemann solver}

In the non-smooth regions, MUSCL-type flux limiting [23] is used for the reconstruction of the left and right states of the conservative fluxes and the advection of the volume fractions. For the conservative problem (4), we employ the HLL Riemann solver introduced by Harten et. al. [24],

$$
F_{j+\frac{1}{2}}= \begin{cases}f\left(\mathbf{u}_{j+\frac{1}{2}}^{L}\right) & \text { if } S_{L} \geq 0 \\ \frac{S_{R} f\left(\mathbf{u}_{j+\frac{1}{2}}^{L}\right)-S_{L} f\left(\mathbf{u}_{j+\frac{1}{2}}^{R}\right)+S_{L} S_{R}\left(\mathbf{u}_{j+\frac{1}{2}}^{R}-\mathbf{u}_{j+\frac{1}{2}}^{L}\right)}{S_{R}-S_{L}} & \text { if } S_{L}<0<S_{R} \\ f\left(\mathbf{u}_{j+\frac{1}{2}}^{R}\right) & \text { if } S_{R} \leq 0\end{cases}
$$

where $S$ denotes the fastest signal velocities. These are taken as estimates of the maximum and minimum eigenvalues of the Jacobian of the flux. In the deterministic case, the eigenvalues of the Jacobian are known analytically, so the method is inexpensive. For the stochastic Galerkin system, analytical expressions are not available, and numerical approximations of the eigenvalues are used instead. In general, obtaining accurate eigenvalue estimates may be computationally costly. However, for the piecewise constant and piecewise linear multiwavelet expansion, we have explicit expressions for the system 
eigenvalues due to the constant eigenvectors of the inner triple product matrices $A$ given by (12), see [25].

The HLL-flux approximates the solution by assuming three states separated by two waves. In the deterministic case, this approximation is known to fail in capturing contact discontinuities and material interfaces [26]. The stochastic Galerkin system is a multiwave generalization of the deterministic case, and similar problems in capturing missing waves are expected. However, by applying flux limiters (minmod) in the same way as in the MUSCL scheme with Roe flux, sharp features of the solution are recovered.

The HLL-flux with MUSCL reconstruction is applied to solve the conservative problem (9). The standard MUSCL scheme is used to solve the advection problem (7) in the regions where the solution is expected to be nonsmooth. In combination with a suitable Runge-Kutta method, the MUSCL scheme is total variation diminshing (TVD) [27]. For the deterministic solution, this would be a sufficient condition for $\alpha$ to attain physically relevant values only. For the stochastic Galerkin system, we need to ensure that the effect of the artificial dissipation from the flux limiters on the different solution modes does not cause the solution $\alpha$ (linear combination of the modes) to become unphysical. By the TVD property, the expectation mode is restricted to $[0,1]$, so unphysical values can only occur if the high order modes are less dissipated than the expectation mode. Artificial dissipation affect highly oscillating functions more than slowly oscillating functions. Since the peaks of the initial functions get sharper with the order of wavelet chaos, the higher order modes are increasingly dissipated by the scheme compared to the lower order modes and the expectation. Thus, most likely the numeri- 
cal volume fraction always remains restricted to physically relevant values as time is evolved.

\subsection{Hybrid scheme}

Numerical interfaces can be designed for stable coupling of problems solved separately using SBP operators. The MUSCL scheme can be rewritten in SBP operator form with an artificial dissipation term [28] and can therefore be coupled with other schemes using SBP operators [20]. In order to enable energy estimates, the artificial dissipation must be zero at the interface.

The computational domain is divided into a left smooth solution region and a right non-smooth solution region that are weakly coupled with an interface. The leftmost lying part of the right region is a transition region where a second order one-sided SBP scheme is applied that transitions into the HLL-MUSCL scheme. In this way, there is a stable coupling between the high-order SBP scheme of the left domain and the second order SBP scheme of the transition region. Numerical dissipation within the order of the scheme is added to the regions where SBP operators are used. Figure 3 schematically depicts the hybrid scheme, applied to two spatial grids that are coupled with an interface.

\subsubsection{An energy estimate for the continuous problem}

We will analyze stability for two solution regions coupled by an interface. However, we start with the continuous problem on a single domain. In order to do this, we symmetrize the two-phase problem. We assume the existence of a convex entropy function $S\left(u^{M}\right)$, i.e. the Hessian $\partial^{2} S / \partial u_{i}^{M} \partial u_{j}^{M}$ 
is positive definite. (Note that convexity as defined here does not allow for zero eigenvalues of the Hessian). Then, by [29], there exists a variable transformation $w^{M}\left(u^{M}\right)=\partial S / \partial u^{M}$ such that $\tilde{f}\left(w^{M}\right)=f\left(u^{M}\right)$ and

$$
\tilde{H} w_{t}^{M}+J_{w} w_{x}^{M}=0
$$

where $w^{M}$ denotes the vector of MW coefficients of the order $M$ approximation of the transformed variables. The inverse Hessian $\tilde{H}=\partial u^{M} / \partial w^{M}=$ $\left(\partial^{2} U / \partial u_{i} \partial u_{j}\right)^{-1}$ and Jacobian $J_{w}=\partial \tilde{f} / \partial w$ are symmetric matrices. Due to convexity $\tilde{H}$ is positive definite and thus defines a norm. As in the case of the Euler equations, the two-phase equations are homogeneous of degree $\tau>-1$, which implies

$$
\tilde{H} w^{M}=\tau u^{M} \quad \text { and } \quad J_{w} w^{M}=\tau \tilde{f}^{M} .
$$

We will use the canonical splittings

$$
u_{t}^{M}=\frac{\tau}{1+\tau} u_{t}^{M}+\frac{1}{1+\tau} \tilde{H} w_{t}^{M}, \quad \tilde{f}_{x}^{M}=\frac{\tau}{1+\tau} \tilde{f}_{x}^{M}+\frac{1}{1+\tau} J_{w} w_{x}^{M} .
$$

To obtain an energy estimate for the continuous problem and stability for the semi-discrete problem, the stochastic Galerkin formulation of the twophase problem must be homogeneous. To show that this holds under the assumption that the corresponding deterministic problem is homogeneous and under some additional assumptions, we consider a deterministic problem that is homogeneous of degree $\tau$. Let

$$
J(u) u=\tau f(u),
$$

with solution $u \in \mathbb{R}^{n}$, Jacobian $J \in \mathbb{R}^{n \times n}$ and flux $f \in \mathbb{R}^{n}$ for a system of $n$ equations. Now assume that the problem satisfying (28) is subject to 
uncertainty in the parameters or in the input conditions. Let $J_{i j}$ denote the $(i, j)$ entry of $J$ which can be expressed as a truncated MW expansion $J_{i j}=\sum_{k=0}^{M}\left(J_{i j}\right)_{k} \psi_{k}$. The stochastic Galerkin Jacobian $\mathcal{J}^{M}$ corresponding to $J$ consists of $n \times n$ submatrices, each of size $(M+1) \times(M+1)$. Let $\mathcal{J}_{i j}^{M}$ be the $(i, j)$ submatrix of $\mathcal{J}^{M}$, defined by $\left[\mathcal{J}_{i j}^{M}\right]_{l m}=\left\langle\psi_{l} \psi_{m} J_{i j}\right\rangle=\sum_{k=0}^{M}\left(J_{i j}\right)_{k}\left\langle\psi_{k} \psi_{l} \psi_{m}\right\rangle, \quad i, j=1, \ldots, n, \quad l, m=0, \ldots, M$

The stochastic Galerkin flux vector of MW coefficients $f^{M}=\left(\left(f_{1}\right)_{0}, \ldots,\left(f_{1}\right)_{M}\right.$, $\left.\ldots,\left(f_{n}\right)_{0}, \ldots,\left(f_{n}\right)_{M}\right)^{T}$ is a nonlinear function and for an arbitrary order $M$ basis of multiwavelets, it is not uniquely defined. To see this, with the pseudo-spectral product $*$ defined in (11), in general

$$
(a * b) * c \neq a *(b * c)
$$

for MW approximations of stochastic functions $a(\xi), b(\xi), c(\xi)$, each one truncated to some order $M$. This implies that the definition of the stochastic Galerkin flux $f^{M}$ depends on the order in which pseudo-spectral operations are performed when evaluating $f^{M}$. Hence, it is not uniquely defined. We may now either restrict ourselves to MW bases where the order of pseudospectral operations does not matter e.g. Haar wavelets, or, we may restrict the order in which pseudo-spectral operations are performed so as to make sure that mathematical properties of interest - e.g. homogeneity - are satisfied. We take the latter approach and define the order $M$ approximation of $f$ through its MW coefficients by

$$
\left(f_{i}\right)_{k} \equiv \frac{1}{\tau} \sum_{j=1}^{n}\left(J_{i j} * u_{j}\right)_{k}, \quad i=1, \ldots, n, \quad k=0, \ldots, M,
$$


which is consistent with the deterministic homogeneous problem. Note that relation (30) is essentially just a restriction on the order of pseudo-spectral operations in the calculation of $f$. It stipulates that $f$ must be defined in terms of the approximation of $J$. Clearly, the approximation of $J$ should also be as close to the true (i.e. inifinite order MW expansion) $J$ as possible. However, for the energy estimates that require homogeneity of the stochastic Galerkin formulation, we only need to satisfy (30).

Proposition 1. Assume that the deterministic problem (28) holds and for a consistent pseudo-spectral approximation $\mathcal{J}^{M}$ of J, let the stochastic Galerkin flux $f^{M}$ be given by the $M W$ coefficients as defined in (30). Then the stochastic Galerkin formulation of order $M$ is also homogeneous of degree $\tau$, i.e. it satisfies

$$
\mathcal{J}^{M}\left(u^{M}\right) u^{M}=\tau f^{M}\left(u^{M}\right)
$$

where $u^{M}=\left(\left(u_{1}\right)_{0}, \ldots,\left(u_{1}\right)_{M}, \ldots,\left(u_{n}\right)_{0}, \ldots,\left(u_{n}\right)_{M}\right)^{T} \in \mathbb{R}^{n(M+1)}$ and $f^{M}=\left(\left(f_{1}\right)_{0}, \ldots,\left(f_{1}\right)_{M}, \ldots,\left(f_{n}\right)_{0}, \ldots,\left(f_{n}\right)_{M}\right)^{T} \in \mathbb{R}^{n(M+1)}$.

Proof. Using the notation (12) for the pseudo-spectral product *, by (29) the $(i, j)$ submatrix of $\mathcal{J}^{M}$ can be written

$$
\mathcal{J}_{i j}^{M}=A\left(J_{i j}^{M}\right), \quad i, j=1, \ldots, n,
$$

where $J_{i j}^{M}=\left(\left(J_{i j}\right)_{0}, \ldots,\left(J_{i j}\right)_{M}\right)^{T}$. Thus, we have

$$
\mathcal{J}^{M}=\left[\begin{array}{ccc}
A\left(J_{11}^{M}\right) & \ldots & A\left(J_{1 n}^{M}\right) \\
\vdots & \ddots & \vdots \\
A\left(J_{n 1}^{M}\right) & \ldots & A\left(J_{n n}^{M}\right)
\end{array}\right]
$$


By the relation (30), any subvector $f_{i}^{M}=\left(\left(f_{i}\right)_{0}, \ldots,\left(f_{i}\right)_{M}\right)^{T}$ of the total flux vector of MW coefficients $f^{M}$ can be written

$$
f_{i}^{M}=\frac{1}{\tau} \sum_{j=1}^{n} A\left(J_{i j}^{M}\right) u_{j}^{M}, \quad i=1, \ldots, n .
$$

Then, considering the $i^{\text {th }}$ row of submatrices,

$$
\left[\mathcal{J}^{M} u^{M}\right]_{i}=\sum_{j=1}^{n} \mathcal{J}_{i j}^{M} u_{j}^{M}=\sum_{j=1}^{n} A\left(J_{i j}^{M}\right) u_{j}^{M}=\tau f_{i}^{M}, \quad i=1, \ldots, n
$$

which is equal to (31).

Remark 2. The original (deterministic) Jacobian entries $J_{i j}$ are nonlinear functions of $u$, and the stochastic Galerkin counterpart $\mathcal{J}^{M}$ is a nonlinear function of the $g P C$ coefficients of $u$. Since the approximation of a nonlinear stochastic function by means of pseudo-spectral operations depends on the order in which the operations are performed, the matrix $\mathcal{J}^{M}$ is not uniquely defined unless we specify the order. However, for the proof of Proposition 1, it is sufficient to define $f^{M}$ as a function of $\mathcal{J}^{M}$, but no need to specify $\mathcal{J}^{M}$ in terms of the order of pseudo-spectral operations.

We will now derive an energy estimate for the continuous symmetrized formulation of the stochastic Galerkin Euler equations in split form,

$$
\frac{\tau}{1+\tau} u_{t}^{M}+\frac{1}{1+\tau} \tilde{H} w_{t}^{M}+\frac{\tau}{1+\tau} \tilde{f}_{x}^{M}+\frac{1}{1+\tau} J_{w} w_{x}=0
$$

Under the conditions of Proposition 1, multiply (32) by $(1+\tau)\left(w^{M}\right)^{T}$ and 
integrate over the physical domain. We get

$$
\begin{gathered}
\tau \int_{0}^{1}\left(w^{M}\right)^{T} u_{t}^{M} d x+\int_{0}^{1}\left(w^{M}\right)^{T} \tilde{H} w_{t}^{M} d x+\tau \int_{0}^{1}\left(w^{M}\right)^{T} \tilde{f}_{x}^{M} d x \\
+\int_{0}^{1}\left(w^{M}\right)^{T} J_{w} w_{x}^{M} d x=\int_{0}^{1}\left(\left(w^{M}\right)^{T}\left(\tilde{H} w^{M}\right)_{t}+\left(w^{M}\right)^{T} \tilde{H} w_{t}^{M}\right) d x \\
+\int_{0}^{1}\left(\left(w^{M}\right)^{T}\left(J_{w} w^{M}\right)_{x}+\left(w^{M}\right)^{T} J_{w} w_{x}^{M}\right) d x=\frac{d}{d t}\left\|w^{M}\right\|_{\tilde{H}}+\left[\left(w^{M}\right)^{T} J_{w} w^{M}\right]_{0}^{1}=0
\end{gathered}
$$

where the first equality follows from (27). The generalized energy estimate (33) is a straightforward stochastic Galerkin generalization of the one given for the deterministic problem in [11].

\subsubsection{Stability in a single domain}

Next we consider the semi-discrete problem and start with a single domain. The stability analysis is a direct generalization of the stability of the symmetrized Euler equations in [11]. We define the flux and the Jacobian under the conditions of Proposition 1 which implies that the stochastic Galerkin system is homogeneous. Let $\mathbf{u}^{M}$ and $\mathbf{w}^{M}$ denote the spatial discretizations of $u^{M}$ and $w^{M}$, respectively, on a mesh consisting of $m$ equidistant points. Let $E_{1}=\operatorname{diag}(1,0, \ldots, 0)$ and $E_{m}=\operatorname{diag}(0, \ldots, 0,1)$. The semi-discretized scheme is

$$
\begin{array}{r}
\frac{\tau}{1+\tau} \mathbf{u}_{t}^{M}+\frac{1}{1+\tau} \hat{H} \mathbf{w}_{t}^{M}+\frac{\tau}{1+\tau}\left(P^{-1} Q \otimes I\right) \tilde{f}^{M}\left(\mathbf{w}^{M}\right)+\frac{1}{1+\tau} \hat{J}_{w}\left(P^{-1} Q \otimes I\right) \mathbf{w}^{M} \\
=\left(P^{-1} E_{1} \otimes \Sigma_{1}^{w}\right)\left(\mathbf{w}^{M}-g_{1}\right)+\left(P^{-1} E_{m} \otimes \Sigma_{m}^{w}\right)\left(\mathbf{w}^{M}-g_{m}\right)
\end{array}
$$

where $\hat{H}$ is block diagonal with each diagonal block equal to $\tilde{H}$ evaluated at the spatial points. $\Sigma_{1}^{w}$ and $\Sigma_{m}^{w}$ are penalty matrices to be determined and 
$g_{1}$ and $g_{m}$ are vectors where only the entries corresponding to the left and right boundary are allowed non-zero values. We assume a diagonal norm $P$, so $(P \otimes I) \hat{H}=\hat{H}(P \otimes I)$. Also, $\hat{J}_{w}$ commutes with $(P \otimes I)$. In order to show stability, we may assume homogeneous boundary conditions $g_{1}=g_{m}=$ 0. Multiplying (34) from the left by $(1+\tau)\left(\mathbf{w}^{M}\right)^{T}(P \otimes I)$ and using the homegeneity properties of (27) yields

$$
\begin{aligned}
\frac{d}{d t}\left\|\mathbf{w}^{M}\right\|_{(P \otimes I) \hat{H}}^{2}+\left(\mathbf{w}^{M}\right)^{T} & \left((Q \otimes I) \hat{J}_{w}+\hat{J}_{w}(Q \otimes I)\right) \mathbf{w}^{M} \\
& =(1+\tau)\left[\left(\mathbf{w}_{1}^{M}\right)^{T} \Sigma_{1}^{w} \mathbf{w}_{1}^{M}+\left(\mathbf{w}_{m}^{M}\right)^{T} \Sigma_{m}^{w} \mathbf{w}_{m}^{M}\right] .
\end{aligned}
$$

Add the transpose of (35) to itself and use the SBP relation (26)

$$
\begin{aligned}
\frac{d}{d t}\left\|\mathbf{w}^{M}\right\|_{(P \otimes I) \hat{H}}^{2}=\left(\mathbf{w}_{1}^{M}\right)^{T}( & \left.J_{w}\left(\mathbf{w}_{1}^{M}\right)+(1+\tau) \Sigma_{1}^{w}\right) \mathbf{w}_{1}^{M} \\
& +\left(\mathbf{w}_{m}^{M}\right)^{T}\left(-J_{w}\left(\mathbf{w}_{m}^{M}\right)+(1+\tau) \Sigma_{m}^{w}\right) \mathbf{w}_{m}^{M} .
\end{aligned}
$$

The scheme is stable with the penalties

$$
\Sigma_{1}^{w}=-\delta_{1} J_{w}^{+}\left(\mathbf{w}_{1}^{M}\right), \quad \Sigma_{m}^{w}=\delta_{m} J_{w}^{-}\left(\mathbf{w}_{m}^{M}\right), \quad \delta_{1}, \delta_{m} \geq \frac{1}{1+\tau} .
$$

Remark 3. The stability analysis above follows that in [11]; for the case $M=0$ the analysis is in fact identical. We show here that the analysis in [11] generalizes to the stochastic Galerkin formulation of order $M$ of multiwavelet expansion under the conditions of Proposition 1.

\subsubsection{Stability at the interface}

Now consider a problem with two domains connected by an interface. By ignoring the imposition of boundary conditions, the semi-discrete systems of 
the left $(L)$ and right $(R)$ domains are given by

$$
\begin{aligned}
\frac{\tau}{1+\tau}\left(\mathbf{u}_{L}^{M}\right)_{t}+\frac{1}{1+\tau} \hat{H}\left(\mathbf{w}_{L}^{M}\right)_{t}+\frac{\tau}{1+\tau}\left(P_{L}^{-1} Q_{L} \otimes I\right) \tilde{f}^{M}\left(\mathbf{w}_{L}^{M}\right) \\
\quad+\frac{1}{1+\tau} \tilde{J}_{w}\left(P_{L}^{-1} Q_{L} \otimes I\right) \mathbf{w}_{L}^{M}=\left(P_{L}^{-1} E_{m} \otimes \Sigma_{L}^{w}\right)\left(\mathbf{w}_{m, L}^{M}-\mathbf{w}_{1, R}^{M}\right),
\end{aligned}
$$

and

$$
\begin{aligned}
\frac{\tau}{1+\tau}\left(\mathbf{u}_{R}^{M}\right)_{t}+\frac{1}{1+\tau} \tilde{J}_{u}\left(\mathbf{w}_{R}^{M}\right)_{t}+\frac{\tau}{1+\tau}\left(P_{R}^{-1} Q_{R} \otimes I\right) \tilde{f}^{M}\left(\mathbf{w}_{R}^{M}\right) \\
\quad+\frac{1}{1+\tau} \tilde{J}_{w}\left(P_{R}^{-1} Q_{R} \otimes I\right) \mathbf{w}_{R}^{M}=\left(P_{R}^{-1} E_{1} \otimes \Sigma_{R}^{w}\right)\left(\mathbf{w}_{1, R}^{M}-\mathbf{w}_{m, L}^{M}\right),
\end{aligned}
$$

respectively. We follow the procedure of section 5.3.2. Multiplying (37) from the left by $(1+\tau)\left(\mathbf{w}_{L}^{M}\right)^{T}\left(P_{L} \otimes I\right)$ and using the homogeneity identity (27), we have

$$
\begin{array}{r}
\frac{d}{d t}\left\|\mathbf{w}_{L}^{M}\right\|_{\left(P_{L} \otimes I\right) \hat{H}}^{2}+\left(\mathbf{w}_{L}^{M}\right)^{T}\left(Q_{L} \otimes I\right) \tilde{J}_{w} \mathbf{w}_{L}^{M}+\left(\mathbf{w}_{L}^{M}\right)^{T} \tilde{J}_{w}\left(Q_{L} \otimes I\right) \mathbf{w}_{L}^{M} \\
=(1+\tau) \mathbf{w}_{m, L}^{M} \Sigma_{L}^{w}\left(\mathbf{w}_{m, L}^{M}-\mathbf{w}_{1, R}^{M}\right) .
\end{array}
$$

Adding the transpose of (39) to itself, neglecting the outer boundaries and performing similar operations on (38), we get

$$
\begin{aligned}
\frac{d}{d t}\left(\left\|\mathbf{w}_{L}^{M}\right\|_{\left(P_{L} \otimes I\right) \hat{H}}^{2}+\left\|\mathbf{w}_{R}^{M}\right\|_{\left(P_{R} \otimes I\right) \hat{H}}^{2}\right) & +\left(\mathbf{w}_{m, L}^{M}\right)^{T} J_{w}\left(\mathbf{w}_{m, L}^{M}\right) \mathbf{w}_{m, L}^{M} \\
-\left(\mathbf{w}_{1, R}^{M}\right)^{T} J_{w}\left(\mathbf{w}_{1, R}^{M}\right) \mathbf{w}_{1, R}^{M}= & (1+\tau)\left(\mathbf{w}_{m, L}^{M}\right)^{T} \Sigma_{L}^{w}\left(\mathbf{w}_{m, L}^{M}-\mathbf{w}_{1, R}^{M}\right) \\
& +(1+\tau)\left(\mathbf{w}_{1, R}^{M}\right)^{T} \Sigma_{R}^{w}\left(\mathbf{w}_{1, R}^{M}-\mathbf{w}_{m, L}^{M}\right) .
\end{aligned}
$$

Assuming symmetric $\Sigma_{L}^{w}$ and $\Sigma_{R}^{w}$, we get the stability condition

$$
\left[\begin{array}{c}
\mathbf{w}_{m, L}^{M} \\
\mathbf{w}_{1, R}^{M}
\end{array}\right]^{T}\left[\begin{array}{cc}
-J_{w}\left(\mathbf{w}_{m, L}^{M}\right)+(1+\tau) \Sigma_{L}^{w} & -\frac{1+\tau}{2}\left(\Sigma_{L}^{w}+\Sigma_{R}^{w}\right) \\
-\frac{1+\tau}{2}\left(\Sigma_{L}^{w}+\Sigma_{R}^{w}\right) & J_{w}\left(\mathbf{w}_{1, R}^{M}\right)+(1+\tau) \Sigma_{R}^{w}
\end{array}\right]\left[\begin{array}{c}
\mathbf{w}_{m, L}^{M} \\
\mathbf{w}_{1, R}^{M}
\end{array}\right] \leq 0 .
$$


Being in the smooth domain we assume $J_{w}\left(\mathbf{w}_{m, L}^{M}\right)=J_{w}\left(\mathbf{w}_{1, R}^{M}\right)=J$ and obtain stability with

$$
\Sigma_{L}^{w}=\frac{1}{1+\tau} J-\theta, \quad \Sigma_{R}^{w}=-\frac{1}{1+\tau} J-\theta,
$$

where $\theta$ is a positive semi-definite matrix. This is completely analogous to the penalties derived for the constant advection problem presented in [20].

The penalties derived in the stability analysis apply to the entropy variables $w$ but in the numerical experiments we use a conservative formulation for correct shock speed and employ the conservative variables $u$. Therefore we need to transform the penalties to the conservative variables. Assuming that the solution is smooth and $\tilde{H}\left(\mathbf{w}_{m, L}^{M}\right)=\tilde{H}\left(\mathbf{w}_{1, R}^{M}\right)$, we rewrite the interface terms

$$
\begin{gathered}
\sum_{L}^{w}\left(\mathbf{w}_{m, L}^{M}-\mathbf{w}_{1, R}^{M}\right)=\frac{1}{1+\tau} J\left(\mathbf{w}_{m, L}^{M}-\mathbf{w}_{1, R}^{M}\right)-\theta\left(\mathbf{w}_{m, L}^{M}-\mathbf{w}_{1, R}^{M}\right) \\
=\frac{1}{1+\tau}\left(\tilde{f}^{M}\left(\mathbf{w}_{m, L}^{M}\right)-\tilde{f}^{M}\left(\mathbf{w}_{1, R}^{M}\right)\right)-\theta \tau\left(\tilde{H}^{-1}\left(\mathbf{w}_{m, L}^{M}\right) \mathbf{u}_{m, L}^{M}-\tilde{H}^{-1}\left(\mathbf{w}_{1, R}^{M}\right) \mathbf{u}_{1, R}^{M}\right) \\
=\frac{1}{1+\tau}\left(f^{M}\left(\mathbf{u}_{m, L}^{M}\right)-f^{M}\left(\mathbf{u}_{1, R}^{M}\right)\right)-\hat{\theta}\left(\mathbf{u}_{m, L}^{M}-\mathbf{u}_{1, R}^{M}\right) \\
=\left(\frac{1}{1+\tau} J_{u}-\hat{\theta}\right)\left(\mathbf{u}_{m, L}^{M}-\mathbf{u}_{1, R}^{M}\right)=\Sigma_{L}^{u}\left(\mathbf{u}_{m, L}^{M}-\mathbf{u}_{1, R}^{M}\right), \quad
\end{gathered}
$$

where

$$
J_{u}=\left.\frac{\partial f^{M}}{\partial u^{M}}\right|_{x=x_{i n t}}
$$

and

$$
\Sigma_{L}^{u}=\frac{1}{1+\tau} J_{u}-\hat{\theta}
$$

and $\hat{\theta}=\tau \theta \tilde{H}^{-1}$ is a positive semi-definite matrix for $\tau>0$ since $\tilde{H}$ is positive definite and $\theta$ is positive semi-definite. Similarly, we get the right penalty 
matrix

$$
\Sigma_{R}^{u}=-\frac{1}{1+\tau} J_{u}-\hat{\theta}
$$

\subsubsection{Conservation at the interface}

In order to show conservation over the interface, we want to mimic the continuous case where we multiply the conservative formulation by a smooth function $\phi$, integrate by parts to get

$$
\int_{0}^{x_{i n t}} \phi u_{t} d x+\int_{x_{i n t}}^{1} \phi u_{t} d x=\int_{0}^{x_{i n t}} \phi_{x} f(u) d x+\int_{x_{i n t}}^{1} \phi_{x} f(u) d x+B . T .
$$

where B.T. denotes outer boundary terms. In (45) no interface terms are present. Consider the semi-discrete scheme

$$
\begin{aligned}
& \left(\mathbf{u}_{L}^{M}\right)_{t}+\left(P_{L}^{-1} Q_{L} \otimes I\right) f^{M}\left(\mathbf{u}_{L}^{M}\right)=\left(P_{L}^{-1} E_{m} \otimes \Sigma_{L}^{u}\right)\left(\mathbf{u}_{m, L}^{M}-\mathbf{u}_{1, R}^{M}\right), \\
& \left(\mathbf{u}_{R}^{M}\right)_{t}+\left(P_{R}^{-1} Q_{R} \otimes I\right) f^{M}\left(\mathbf{u}_{R}^{M}\right)=\left(P_{R}^{-1} E_{1} \otimes \Sigma_{R}^{u}\right)\left(\mathbf{u}_{1, R}^{M}-\mathbf{u}_{m, L}^{M}\right)
\end{aligned}
$$

Multiplying from the left by $\boldsymbol{\phi}_{L}^{T}\left(P_{L} \otimes I\right)$ and $\boldsymbol{\phi}_{R}^{T}\left(P_{R} \otimes I\right)$, respectively, where $\phi_{L}$ and $\phi_{L}$ are discretized smooth functions satisfying $\phi_{m, L}=\phi_{1, R}=\phi_{I}$, we get

$$
\begin{aligned}
& \boldsymbol{\phi}_{L}^{T}\left(P_{L} \otimes I\right)\left(\mathbf{u}_{L}^{M}\right)_{t}+\boldsymbol{\phi}_{R}^{T}\left(P_{R} \otimes I\right)\left(\mathbf{u}_{R}^{M}\right)_{t}=\left(D_{L} \boldsymbol{\phi}_{L}\right)^{T}\left(P_{L} \otimes I\right) f^{M}\left(\mathbf{u}_{L}^{M}\right) \\
&+\left(D_{R} \boldsymbol{\phi}_{R}\right)^{T}\left(P_{R} \otimes I\right) f^{M}\left(\mathbf{u}_{R}^{M}\right)+B . T . \\
&+\boldsymbol{\phi}_{I}^{T}\left[\left(\mathbf{u}_{m, L}^{M}-\mathbf{u}_{1, R}^{M}\right)\left(\Sigma_{L}^{u}-\Sigma_{R}^{u}\right)-f^{M}\left(\mathbf{u}_{m, L}^{M}\right)+f^{M}\left(\mathbf{u}_{1, R}^{M}\right)\right] .
\end{aligned}
$$

The semi-discrete formulation (48) mimics the continuous expression (45) if we choose $\Sigma_{L}^{u}$ and $\Sigma_{R}^{u}$ such that

$$
\left(\mathbf{u}_{m, L}^{M}-\mathbf{u}_{1, R}^{M}\right)\left(\Sigma_{L}^{u}-\Sigma_{R}^{u}\right)-f^{M}\left(\mathbf{u}_{m, L}^{M}\right)+f^{M}\left(\mathbf{u}_{1, R}^{M}\right)=0
$$


We assume $J_{u}\left(\mathbf{u}_{m, L}^{M}\right)=J_{u}\left(\mathbf{u}_{1, R}^{M}\right)=J_{u}$. Then, the interface terms cancel with the choice $\Sigma_{L}^{u}-\Sigma_{R}^{u}=J_{u}$, which is consistent with the condition for stability given by the penalties (43) and (44) and $\tau=1$.

\section{Numerical results}

The exact solution of the test problem is known analytically for any given value of the stochastic variable $\xi$. Thus, we can obtain the exact statistics to arbitrary accuracy by averaging the exact Riemann solutions over a large number of realizations of $\xi$. In the numerical experiments, we will assume $\xi \sim$ $\mathcal{U}[-0.05,0.05]$, where $\mathcal{U}$ denotes the uniform distribution. For the numerical solutions, we use SBP operators that can be found in [30].

\subsection{Convergence of smooth solutions}

The method of manufactured solutions is used to impose a smooth time dependent solution of the two-phase problem through a source term. We consider the manufactured solution defined by

$$
\begin{aligned}
& \alpha=\alpha_{0}+\alpha_{1} \tanh \left(s\left(x_{0}-x+t+\xi\right)\right) \\
& \beta=\beta_{0}+\beta_{1} \tanh \left(-s\left(x_{0}-x+t+\xi\right)\right) \\
& v=\tanh \left(s\left(v_{0}+x_{0}-x+t+\xi\right)\right)+\tanh \left(-s\left(-v_{0}+x_{0}-x+t+\xi\right)\right) \\
& p=p_{0}+p_{1} \tanh \left(s\left(x_{0}-x+t+\xi\right)\right),
\end{aligned}
$$

with $s=15, v_{0}=0.03, \alpha_{0}=\alpha_{1}=\beta_{0}=\beta_{1}=0.5, p_{0}=0.75, p_{1}=0.25$. We take $\rho_{\alpha}=1$ and $\rho_{\beta}=0.125$. We measure the error in the $L_{2}(\Omega, \mathcal{P})$ norm and 
the discrete $\ell_{2}$ norm,

$$
\begin{aligned}
\left\|u^{M}-u\right\|_{2,2} & \equiv\left\|u^{M}-u\right\|_{\ell_{2}, L_{2}(\Omega, \mathcal{P})}=\left(\Delta x \sum_{i=1}^{m}\left\|u^{M}\left(x_{i}, t, \xi\right)-u\left(x_{i}, t, \xi\right)\right\|_{L_{2}(\Omega, \mathcal{P})}^{2}\right)^{1 / 2} \\
= & \left(\Delta x \sum_{i=1}^{m} \int_{\Omega}\left(u^{M}\left(x_{i}, t, \xi\right)-u\left(x_{i}, t, \xi\right)\right)^{2} d \mathcal{P}(\xi)\right)^{1 / 2}= \\
& \approx\left(\Delta x \sum_{i=1}^{m} \sum_{j=1}^{q}\left(u^{M}\left(x_{i}, t, \xi_{q}^{(j)}\right)-u\left(x_{i}, t, \xi_{q}^{(j)}\right)\right)^{2} w_{q}^{(j)}\right)^{1 / 2}
\end{aligned}
$$

where a $q$-point quadrature rule with points $\left\{\xi_{q}^{(j)}\right\}_{j=1}^{q}$ and weigths $\left\{w_{q}^{(j)}\right\}_{j=1}^{q}$ was used in the last line to approximate the integral in $\xi$. The GaussLegendre quadrature is used here since the solution is smooth in the stochastic dimension.

Figure 4 (a) shows the spatial convergence when the proportion of loworder and high-order points remains constant. The low-order scheme dominates the error, so the overall convergence rate is second order. In regions of fourth order operators, the error levels are lower and therefore the local accuracy higher compared to the regions of second order operators, see Figure 4 (b). This is further illustrated in Figure 5 where a similar problem with sharp gradients in the middle of the domain is solved with a hybrid scheme where fourth order operators are used for the region of large gradients and second order operators are used for the regions next to the boundaries. With constant proportion of high order points under mesh refinement, the convergence is second order. The comparison with the solution with second order operators throughout the computational domain, also included in Figure 5, shows that the error of the the hybrid scheme is smaller.

Figure 6 (a) shows the spatial convergence employing three computational 
domains separated by two interfaces. The middle domain is solved with second order SBP and the left and right domains with fourth order SBP. The number of points in the second order region remains constant (20 points), as the high order domains are refined. Figure 6 (b) depicts the spatial convergence with three domains, all solved with fourth order SBP. The proportion of points in each region remains the same so the interface locations do not change when the grids are refined.

\subsection{Non-smooth Riemann problem}

With the hybrid scheme as depicted schematically in Figure 3, we solve the problems (7)-(9) with the boundary conditions in (6) and assuming $\xi \sim \mathcal{U}[-0.05,0.05]$. Figure 7 shows the variances of density, velocity, energy and pressure at $t=0.05$. The error from the interface is not significant compared to the error due to the stochastic truncation and spatial resolution. A relatively fine mesh and high order MW expansion is required to capture the variance of the solution. Especially high order MW coefficients exhibit sharp spatial variation. Thus, to attain a given level of accuracy, more spatial grid points are required for the stochastic Galerkin problem compared to the deterministic problem.

Figure 8 depicts the convergence of pressure statistics with increasing order of MW on a fixed spatial grid of 400 points. In the analysis of regularity in Section 4, we anticipated the solution to develop a larger number of weaker discontinuities as the order of MW expansion increases. This behavior can be observed in Figure 8. All (visible) discontinuities are located in the right domain where the shock-capturing method is used. 


\section{Conclusions}

In order to efficiently solve fluid flow problems, a feasible strategy is to locally adapt the numerical method to the smoothness of the solution whenever these properties are known or can be estimated. A two-phase Riemann problem with uncertain initial discontinuity location has been investigated with respect to the smoothness properties of the MW coefficients of the solution. Whereas the corresponding deterministic problem has a discontinuous solution profile, the stochastic modes of the gPC expansion of the true solution are smooth.

A symmetrization and combination of conservative and non-conservative formulation leads to a generalized energy estimate for the stochastic Galerkin system, just as for the case of the deterministic Euler equations. Under certain smoothness assumptions, stability at the interfaces can be obtained for the symmetrized system. The derived penalty matrices are transformed back to the conservative variable formulation that is used in the numerical experiments.

The numerical results show that the convergence rate for the smooth problem (smoothness enforced by the method of manufactured solutions) is second order when fourth order and second order operators are combined and the proportion of second order points remains constant during mesh refinement. However, the error is smaller in this case compared to the case of a single domain solved with second order operators.

The two-phase non-smooth Riemann problem is reasonably well resolved

with the hybrid scheme combining high order SBP operators in the smooth regions with the HLL solver and MUSCL reconstruction in the spatial region 
containing discontinuities. A relatively large number of multiwavelets are needed to accurately represent the stochastic solution. This in turn requires a fine spatial mesh for accurate resolution.

The framework presented here can be extended to time-dependent interfaces that are adapted to the evolving regions of non-smooth solutions. A moving mesh based on interfaces and SBP-operators has already been designed for deterministic problems in [20] and this technique could be used for stochastic Galerkin systems. Depending on the problem, different MW bases can be used in the different spatial regions for efficient representation of the local uncertainty.

In the case of a moving interface, several detection strategies can be used relying either on the physical solution, e.g. $\alpha=0.5$, or on the overall variance which would provide a measure of the oscillations. Further investigations are required to identify the most effective detection algorithm.

\section{Acknowledgements}

Financial support has been provided by the German Research Foundation (Deutsche Forschungsgemeinschaft - DFG) in the framework of the Sonderforschungsbereich Transregio 40 and the IGSSE (International Graduate School of Science and Engineering) at the TU München. The authors would like to thank Dr. Xiangyu Hu, Kwok Kai So and Dr. Anna Nissen for ideas and fruitful discussions regarding the problem formulation and numerical methods.

[1] K. K. So, T. Chantrasmi, X. Y. Hu, J. A. S. Witteveen, C. Stemmer, G. Iaccarino, N. A. Adams, Uncertainty analysis for shock-bubble in- 
teraction, in: Proceedings of the 2010 Summer Program, Center for Turbulence Research, Stanford University, USA.

[2] J.-F. Haas, B. Sturtevant, Interaction of weak shock waves with cylindrical and spherical gas inhomogeneities, Journal of Fluid Mechanics, 181(1987) 41-76.

[3] H. Li, D. Zhang, Efficient and Accurate Quantification of Uncertainty for Multiphase Flow With the Probabilistic Collocation Method, SPE J., 14(2009) 665-679.

[4] M. Chen, A. A. Keller, Z. Lu, D. Zhang, G. Zyvoloski, Uncertainty Quantification for Multiphase Flow in Random Porous Media Using KLBased Moment Equation Approaches, AGU Spring Meeting Abstracts (2008) A2.

[5] D. Xiu, G. E. Karniadakis, The Wiener-Askey Polynomial Chaos for Stochastic Differential Equations, SIAM J. Sci. Comput. 24 (2002) 619644.

[6] M. K. Deb, I. M. Babuska, J. T. Oden, Solution of stochastic partial differential equations using Galerkin finite element techniques, Computer Methods in Applied Mechanics and Engineering 190 (2001) 6359 - 6372.

[7] C. L. Pettit, P. S. Beran, Spectral and multiresolution Wiener expansions of oscillatory stochastic processes, Journal of Sound and Vibration 294 (2006) 752-779.

[8] Q.-Y. Chen, D. Gottlieb, J. S. Hesthaven, Uncertainty analysis for the 
steady-state flows in a dual throat nozzle, J. Comput. Phys. 204 (2005) 378-398.

[9] P. Pettersson, G. Iaccarino, J. Nordström, Numerical analysis of the Burgers' equation in the presence of uncertainty, J. Comput. Phys. 228 (2009) 8394-8412.

[10] C. Schwab, S. A. Tokareva, High order approximation of probabilistic shock profiles in hyperbolic conservation laws with uncertain initial data, Technical Report 2011-53, ETH, Zürich, 2011.

[11] M. Gerritsen, P. Olsson, Designing an efficient solution strategy for fluid flows, J. Comput. Phys. 129 (1996) 245-262.

[12] M. H. Carpenter, J. Nordström, D. Gottlieb, A stable and conservative interface treatment of arbitrary spatial accuracy, J. Comput. Phys. 148 (1999) 341-365.

[13] R. G. Ghanem, P. D. Spanos, Stochastic finite elements: a spectral approach, Springer-Verlag, New York, 1991.

[14] O. P. Le Maître, O. M. Knio, H. N. Najm, R. G. Ghanem, Uncertainty propagation using Wiener-Haar expansions, J. Comput. Phys. 197 (2004) 28-57.

[15] O. P. Le Maître, O. M. Knio, Spectral Methods for Uncertainty Quantification, Springer, first edition, 2010.

[16] O. P. Le Maître, H. N. Najm, R. G. Ghanem, O. M. Knio, Multi- 
resolution analysis of Wiener-type uncertainty propagation schemes, J. Comput. Phys. 197 (2004) 502-531.

[17] B. K. Alpert, A class of bases in L2 for the sparse representations of integral operators, SIAM J. Math. Anal. 24 (1993) 246-262.

[18] B. J. Debusschere, H. N. Najm, P. P. Pébay, O. M. Knio, R. G. Ghanem, O. P. Le Maitre, Numerical Challenges in the Use of Polynomial Chaos Representations for Stochastic Processes, SIAM J. Sci. Comput. 26 (2005) 698-719.

[19] G. A. Sod, A survey of several finite difference methods for systems of nonlinear hyperbolic conservation laws, J. Comput. Phys. 27 (1978) $1-31$.

[20] S. Eriksson, Q. Abbas, J. Nordström, A stable and conservative method for locally adapting the design order of finite difference schemes, J. Comput. Phys. 230 (2011) 4216-4231.

[21] H.-O. Kreiss, G. Scherer, Finite element and finite difference methods for hyperbolic partial differential equations, in: Mathematical Aspects of Finite Elements in Partial Differential Equations, Academic Press, New York, 1974, 179-183.

[22] B. Strand, Summation by parts for finite difference approximations for d/dx, J. Comput. Phys. 110 (1994) 47-67.

[23] B. van Leer, Towards the ultimate conservative difference scheme. V - A second-order sequel to Godunov's method, J. Comput. Phys. 32 (1979) $101-136$. 
[24] A. Harten, P. D. Lax, B. van Leer, On Upstream Differencing and Godunov-Type Schemes for Hyperbolic Conservation Laws, SIAM Review 25 (1983) 35-61.

[25] P. Pettersson, G. Iaccarino, J. Nordström, A Stochastic Galerkin Method for the Euler Equations with Roe Variable Transformation, Technical Report 2012-033, Department of Information Technology, Uppsala University, 2012.

[26] E. F. Toro, Riemann Solvers and Numerical Methods for Fluid Dynamics: A Practical Introduction, Springer, Berlin, second edition, 1999.

[27] S. Gottlieb, C.-W. Shu, Total variation diminishing Runge-Kutta schemes, Math. Comp. 67 (1998) 73-85.

[28] Q. Abbas, E. van der Weide, J. Nordström, Accurate and stable calculations involving shocks using a new hybrid scheme, in: Proc. 19th AIAA CFD Conference, number 2009-3985 in Conference Proceeding Series, AIAA, 2009.

[29] A. Harten, On the symmetric form of systems of conservation laws with entropy, J. Comput. Phys. 199 (2004) 503-540.

[30] K. Mattsson, J. Nordström, Summation by parts operators for finite difference approximations of second derivatives, J. Comput. Phys. 199 (2004) 503-540. 

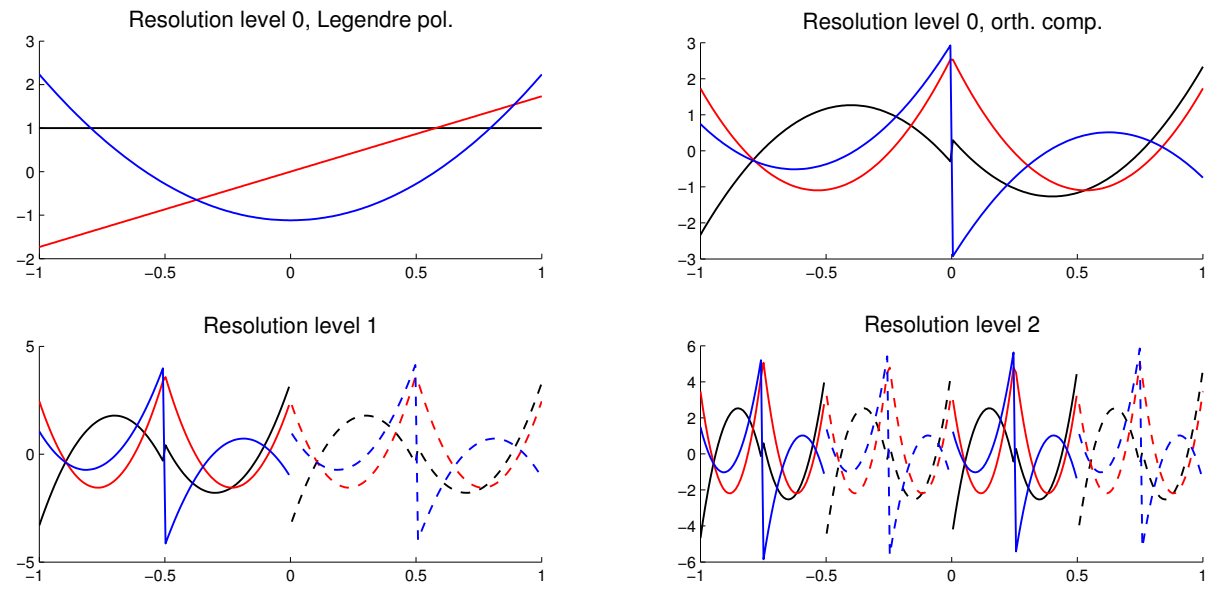

Figure 1: Multiwavelets for $N_{p}=2, N_{r}=3$. Resolution level 0 consists of the first $N_{p}+1$ Legendre polynomials and their orthogonal complement. Resolution level $j>0$ contains $\left(N_{p}+1\right) 2^{j}$ wavelets each. Each basis function is a piecewise polynomial of order $N_{p}$.
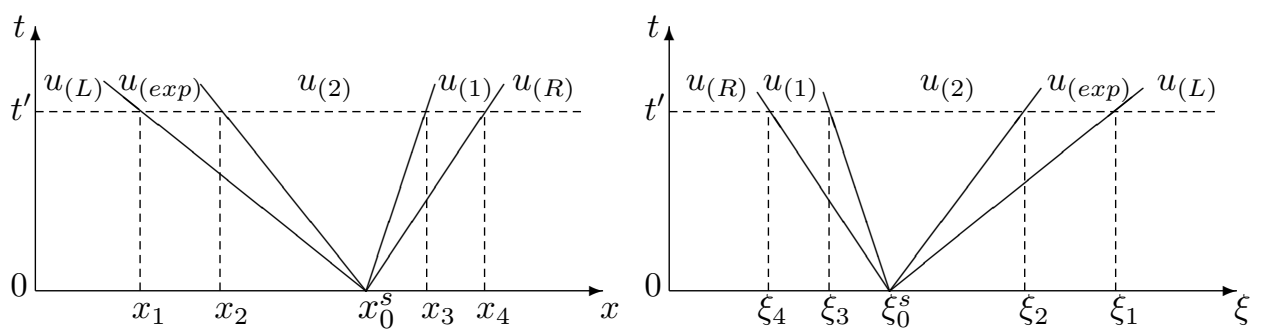

Figure 2: Schematic representation of the solution of the twophase problem. Solution regions in the $x-t$ space for a fixed $\xi$ (left), and solution regions in $\xi-t$ space for a fixed $x$ (right). 


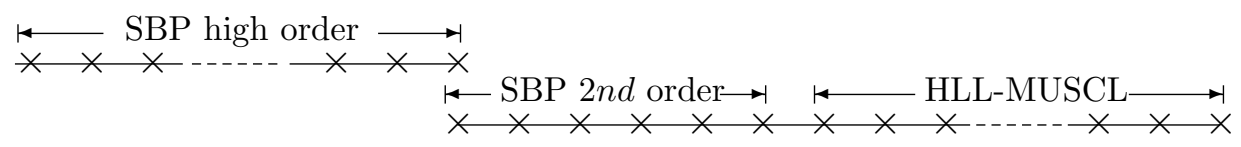

Figure 3: Discretization methods for the different regions of the spatial mesh. The point at the interface is represented in both of the adjacent schemes.
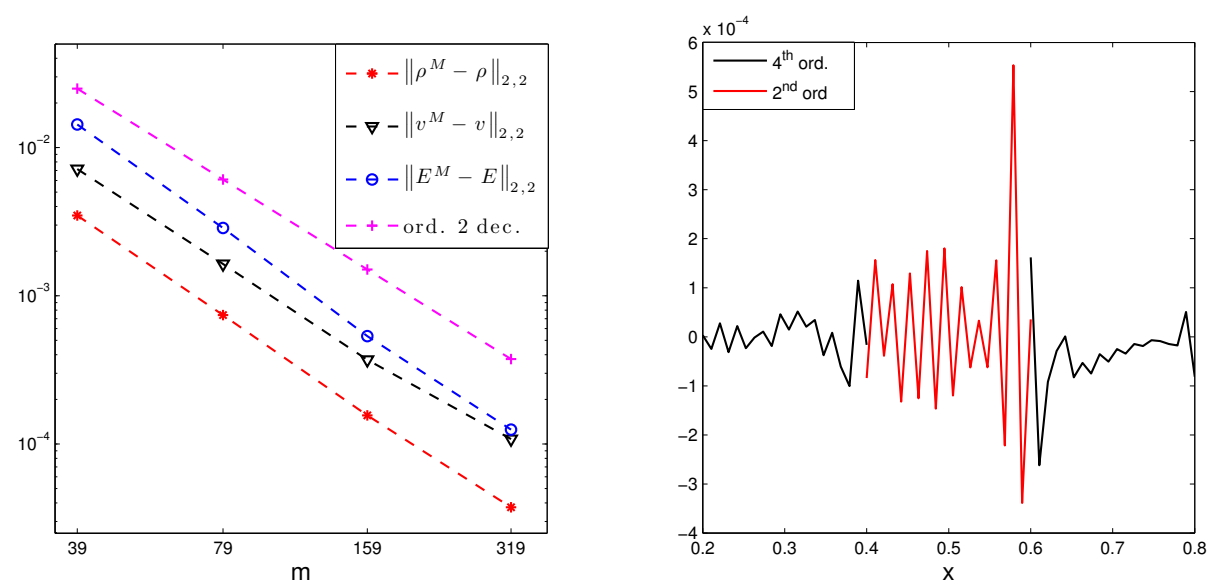

(a) Norm of errors (defined in (49)) for (b) Absolute error in mean density, $t=$ smooth solution, $t=0.05$.

$0.1,60$ spatial points.

Figure 4: SBP 4-2-4, fixed proportion of SBP 2 points. $N_{p}=8$, $N_{r}=0$ order of multiwavelets (Legendre polynomials). 


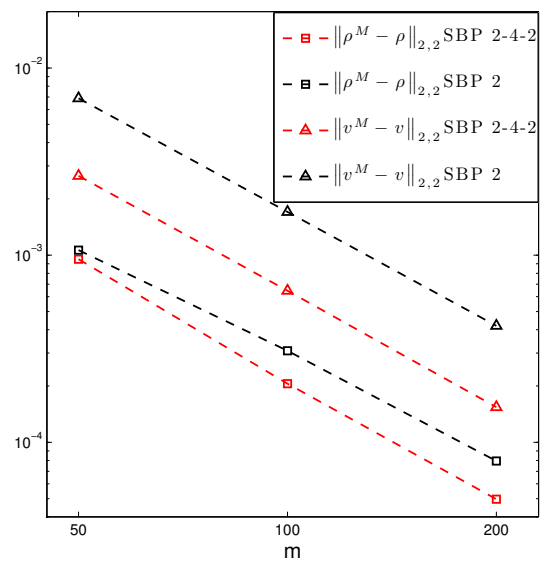

Figure 5: Comparison of norm of errors (defined in (49)), three solution regions SBP 2-4-2 versus single region solved with SBP 2, $t=0.1$. The proportion of fourth order points remains constant during mesh refinement. $N_{p}=8, N_{r}=0$ order of multiwavelets (Legendre polynomials). 

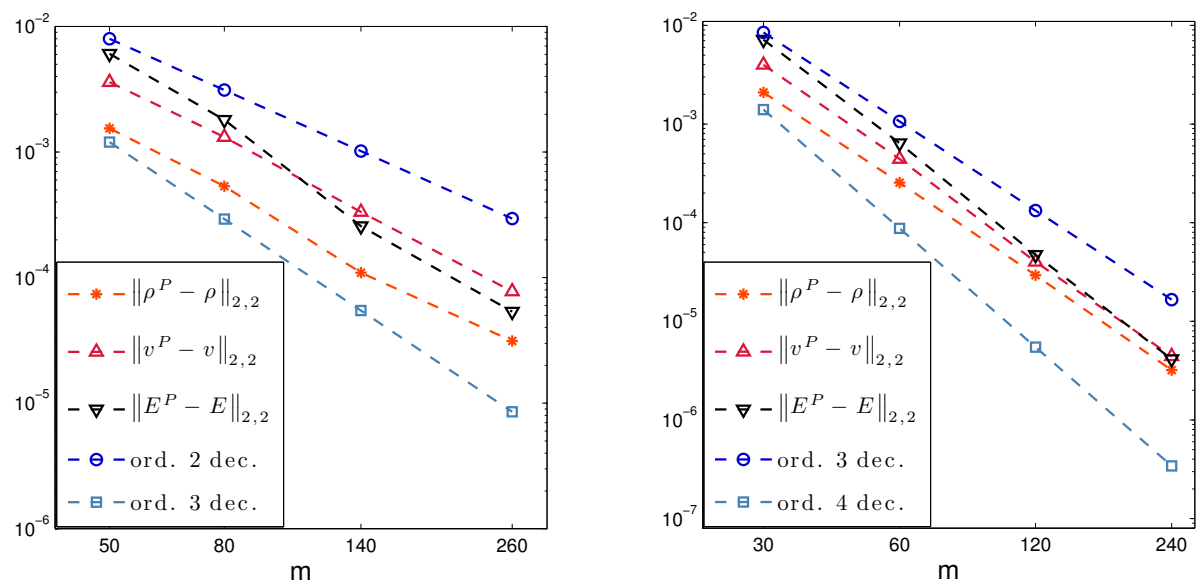

(a) Norm of errors, SBP4-SBP2-SBP4, (b) Norm of errors, three SBP4 schemes fixed number of SBP2 points. coupled by two interfaces.

Figure 6: Spatial convergence with three regions and two interfaces. $t=0.05 . N_{p}=8, N_{r}=0$ order of multiwavelets (Legendre polynomials). 

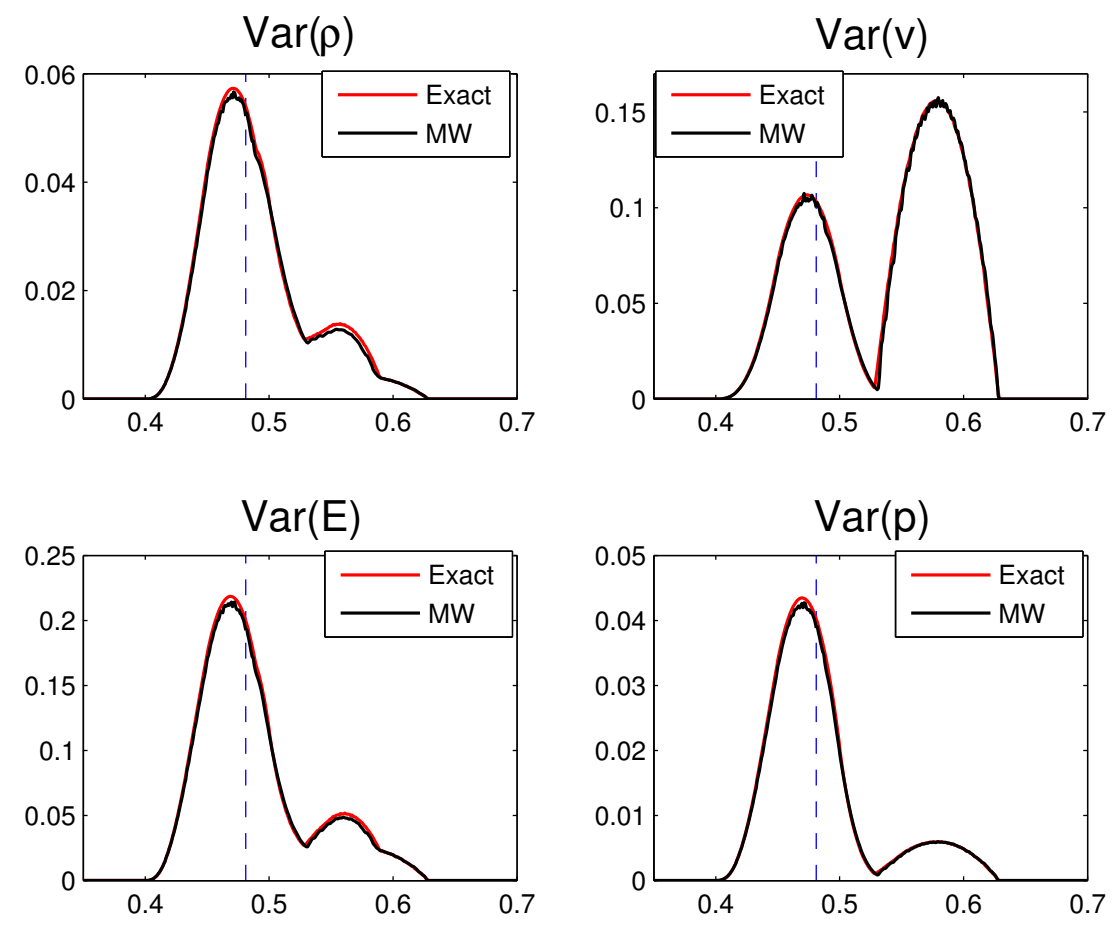

Figure 7: Variances at $t=0.05, m=400$, fourth order SBP (left region) single interface (dashed blue line) and HLL-MUSCL (right region), $\left(N_{p}, N_{r}\right)=(0,5)$ (Haar wavelets). 


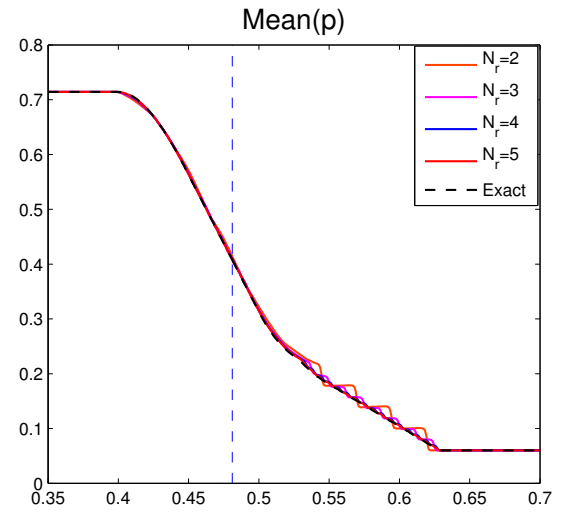

(a) Mean pressure.

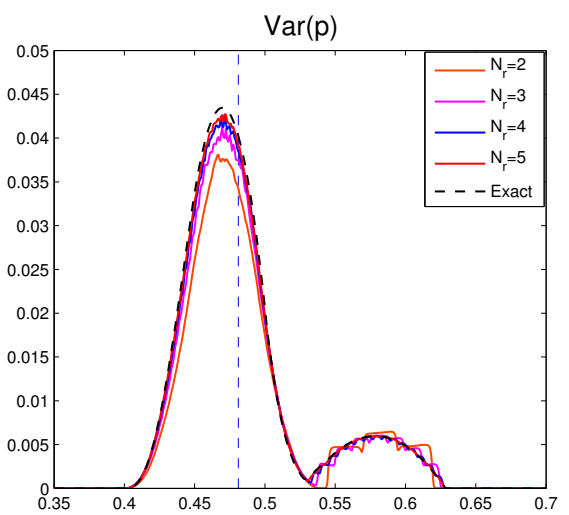

(c) Variance of pressure.

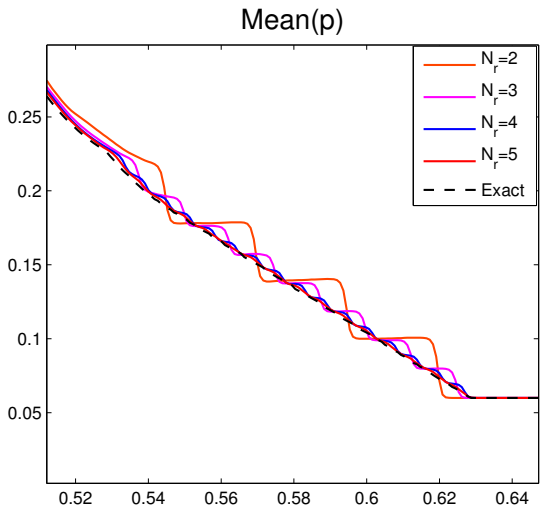

(b) Mean pressure in the proximity of the deterministic shock.

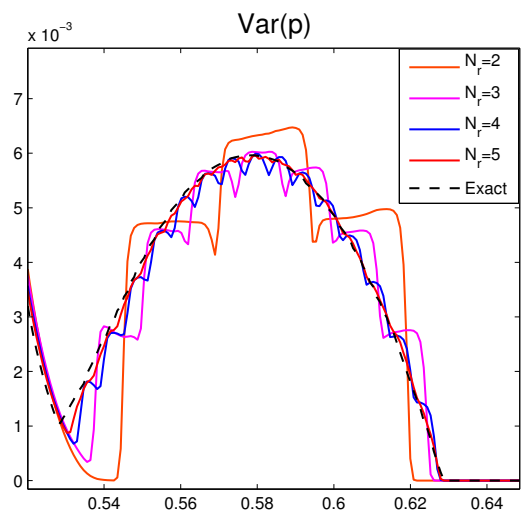

(d) Variance of pressure in the proximity of the deterministic shock.

Figure 8: Convergence of the mean and variance of pressure with the order of MW chaos, different orders of piecewise constant MW. $t=0.05, m=400$. Fourth order SBP (left domain) and HLL-MUSCL (right domain). The vertical dashed line indicates the location of the numerical interface. 